\title{
Plexiform Angiomyxoid Myofibroblastic Tumor of the Stomach
}

\author{
Peifeng Li ${ }^{1,2}$, Qingqing Zhang ${ }^{3}$, Xuchun $\mathrm{Jia}^{1}$, Qinlong $\mathrm{Li}^{1}$, Zengshan $\mathrm{Li}^{1}$, Zhe Wang ${ }^{{ }^{*}}$ \\ ${ }^{1}$ State Key Laboratory of Cancer Biology, Department of Pathology, Xijing Hospital and School of Basic Medicine, Fourth Military \\ Medical University, Xi'an, China; ${ }^{2}$ Department of Pathology, The General Hospital of Jinan Military Command, Ji'nan, China; \\ ${ }^{3}$ Department of Hepatobiliary Surgery, Weifang People’s Hospital, Weifang, China. \\ Email: *zhwang@fmmu.edu.cn
}

Received June 22 ${ }^{\text {nd }}$, 2012; revised July $13^{\text {th }}, 2012$; accepted July $23^{\text {rd }}, 2012$

\begin{abstract}
Plexiform angiomyxoid myofibroblastic tumor (PAMT) is a recently described gastric tumor with a peculiar plexiform pattern, bland spindle cells and a myxoid stroma rich in arborizing blood vessels. PAMT of the stomach is a very rare tumor without distinctive clinical manifestations. In this study, we report a new case of PAMT which is the first Chinese case in English literature. A 47-year-old Chinese woman was admitted with a 6-month history of intermittent epigastric discomfort, and abdominal pain for 2 months. Gastroscopy showed an elevated mass in the anterior wall of the gastric antrum. Endoscopic ultrasound revealed a focal hypoechoic lesion protruding into the lumen. A laparoscopic distal gastrectomy was performed, and the patient made an uneventful recovery and remains well 1.5 years later. A diagnosis of PAMT was made by histopathology and immunochemistry.
\end{abstract}

Keywords: Plexiform Angiomyxoid Myofibroblastic Tumor; Stomach; Gastrointestinal Stromal Tumor; Myofibroblast

\section{Introduction}

Plexiform angiomyxoid myofibroblastic tumor (PAMT) is a recently described gastric tumor with a peculiar plexiform pattern, bland spindle cells and a myxoid stroma rich in blood vessels [1]. It almost exclusively occurs in the gastric antrum. The myofibroblastic-fibroblastic nature of the tumor cells has been confirmed by immunohistochemical and ultrastructural analyses [2]. There have been 23 reported cases of gastric PAMT to date [3]. This is a rare tumor with equal gender distribution occurring primarily in adults with a wide range of 7 to 75 years [4]. Clinical symptoms are related to ulceration of the mucosa from the underlying lesions, so hematemesis and anemia are most commonly encountered. Here we report an additional case of PAMT of the stomach. Our current case shares the clinical, histological, immunophenotypical features of the previously described cases.

\section{Case Report}

A 47-year-old Chinese woman was admitted with a 6-month history of intermittent epigastric discomfort, and abdominal pain for 2 months. Gastroscopy showed an elevated mass with a smooth surface measuring ap-

${ }^{*}$ Corresponding author. proximately $3.5 \mathrm{~cm} \times 3.5 \mathrm{~cm}$ in the anterior wall of the gastric antrum (Figure 1(a)). Endoscopic ultrasound revealed a focal hypoechoic lesion protruding into the lumen, mainly in the submucosa and muscularis propria (Figure 1(b)). A laparoscopic distal gastrectomy was performed, and the patient made an uneventful recovery and remains well 1.5 years later.

Gross examination of the stomach showed a well-circumscribed polypoidal tumor measuring $5.0 \mathrm{~cm} \times 3.0 \mathrm{~cm}$ $\times 2.0 \mathrm{~cm}$ in the anterior antral wall. Cut section revealed a solid, glistening translucid tumor mainly in the sub-

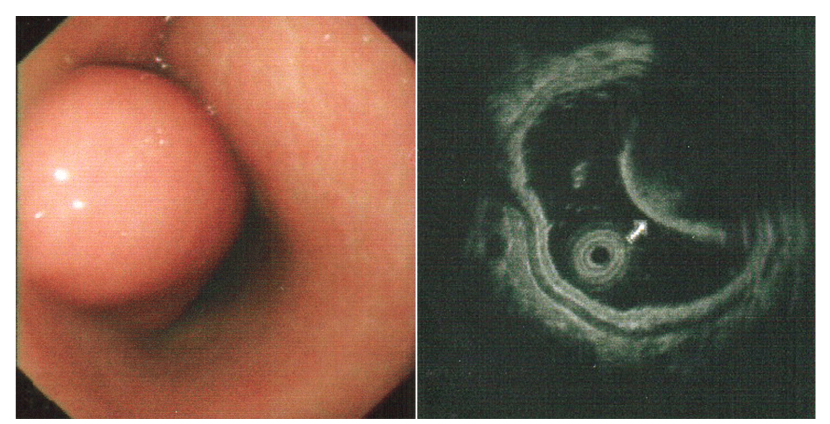

(a)

(b)

Figure 1. PAMT imaging in the stomach. (a) Gastroscopy showing an elevated mass in the anterior wall of the gastric antrum; (b) Endoscopic ultrasound showing a focal hypoechoic lesion protruding into the lumen. 
mucosa, poorly demarcated from the muscularis propria, and with mucoid areas (Figure 2(a)). The serosal surface was studded with multiple polypoid tumor projections. Histological examination showed extension from the submucosa to the serosa. The tumor exhibited an irregular multinodular plexiform pattern (Figure 2(b) and (c)). The cells were spindle-shaped, with no significant nuclear atypia or mitosis, and were disposed randomly or in a vague fascicular fashion, separated by an abundant myxoid extracellular matrix that was alcian blue ( $\mathrm{pH} 2.5)$ positive and in which a network of fine capillary-caliber arborizing vessels was observed. Stromal collagenization was also noted. Mast cells were scattered in the stroma, but infiltration by lymphocytes, plasma cells and eosinophils was inconspicuous. Tumor necrosis was not observed. Immunohistochemically, the tumor cells were diffusely positive for $\alpha$-smooth muscle actin (SMA) (Figure 2(d)), but negative for CD117, CD34, S-100 protein, anaplastic lymphoma kinase (ALK), $\beta$-catenin, and H-caldesmon. The Ki-67 labeling index was less than $1 \%$. Based on the histological features, and supported by the immunostaining findings, a diagnosis of PAMT was made. This study was reviewed and approved by our ethics committee.

\section{Discussion}

Takahashi et al. described 2 cases of a unique gastric mesenchymal tumor designated as "plexiform angiomyxoid myofibroblastic tumor (PAMT)” in 2007 [2]. Two years later, Miettinen et al. described a series of similar tumors, and they advocated the use of the appellation "plexiform fibromyxoma" [5]. Typical histological features of this entity include multinodular plexiform growth pattern, spindle-shape bland myofibroblastic tumor cells (positive for a-SMA) and myxoid matrix that is rich in small vessels, but fibrosis or collagenous matrix is only observed in some cases. Therefore, we believe PAMT is an appropriate diagnostic term to cover histo-
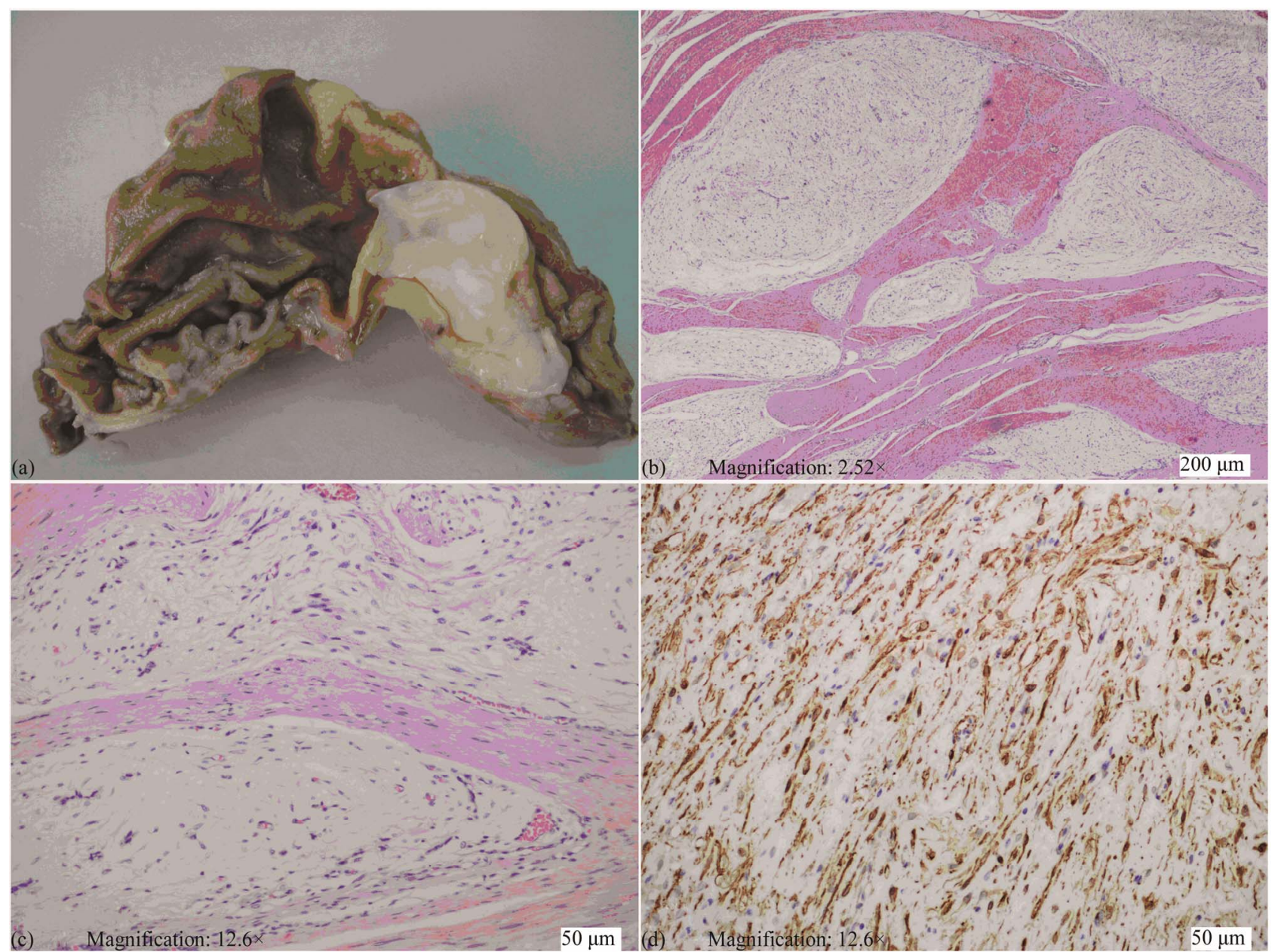

Figure 2. Pathological features of PAMT. (a) Cut section of PAMT showing a solid glistening translucid tumor; (b) Histological finding exhibiting an multinodular plexiform pattern with bland spindle tumor cells (H \& $E$ stains, original magnification $\times 40$ ), myxoid extracellular matrix and fine arborizing vessels (c) (H \& E stains, original magnification $\times 200)$; (d) Tumor cells diffusely positive for SMA (IHC stains, original magnification $\times 200$ ). 
genesis and histology. However, the WHO classification of tumors of the digestive system accepted "plexiform fibromyxoma” as a diagnostic term instead of PAMT [6].

PAMT of the stomach is a very rare tumor without distinctive clinical manifestations. Symptoms may include those of ulceration, hematemesis and anemia. Our patient presented with intermittent epigastric discomfort and abdominal pain. The endoscopist usually encounters a submucosal-based or nodular mass. A very striking feature is the almost exclusive location in the gastric antrum. The mucosa may be intact, dimpled or ulcerated.

Microscopic examination reveals a uniform spindle cell population arranged in a plexiform multinodular pattern. Individual tumor cells have bland oval nuclei and weakly eosinophilic cytoplasm. The stroma is also typical and ranges from being myxoid to fibromyxoid to hyalinized. The vasculature within the stroma is prominent and varies from small thin-walled vascular channels to more ectatic arborizing vessels. Cytological atypia, necrosis, and a brisk mitotic rate are not seen. Immunohistochemistry shows PAMT to be smooth muscle actin positive, focal desmin, and caldesmon positive but negative for CD117, CD34, S-100 protein, neurofilament, cytokeratins, epithelial membrane antigen, and ALK.

Although PAMT demonstrates characteristic pathological features, it should be considered in the differential diagnosis of gastrointestinal stromal tumors (GIST) and other mesenchymal tumors of the stomach, such as leiomyoma, schwannoma, perineurioma, fibromatosis, solitary fibrous tumor, inflammatory fibroid polyp, and inflammatory myofibroblastic tumor. The application of an appropriate panel of antibodies and awareness of PAMT should result in the correct diagnosis being made [3]. GIST does not show the distinctive plexiform intramural growth pattern and are typically positive for CD117 or DOG1. Myxoid leiomyoma is positive for SMA, desmin and caldesmon and plexiform neurofibroma is positive for S-100 protein. Inflammatory fibroid polyp is usually small submucosal lesion composed of epithelioid to spindled fibroblasts and inflammatory cells.

Due to its rarity, the true biological potential of PAMT remains unknown. However, the bland nuclear features, low proliferative index and absence of necrosis, vascular invasion, recurrence or metastasis in all PAMT cases reported to date justify its characterization as a benign tumor. Currently, complete excision remains the treatment of choice.

\section{Conclusion}

PAMT is a very rare stomach tumor of mesenchymal origin. The typical histologic features of PAMT include multinodular plexiform growth pattern, spindle-shaped tumor cells, myxoid stroma, and abundant blood vessels. Immunohistochemical findings suggested that the tumor cells were myofibroblastic in nature, with positive reactions for SMA, but negative reactions for CD34, CD117, S-100 protein, ALK, $\beta$-catenin, and $\mathrm{H}$-caldesmon. The Ki-67 labeling index was less than 1\%. PAMT is a distinctive benign gastric antral neoplasm that should be separated from GIST, nerve sheath tumors, and other fibromyxoid neoplasms. When myxoid spindle cell lesion is observed in endoscopic biopsy, PAMT should be included in diferential diagnosis.

\section{Acknowledgements}

This work is supported in part by National Natural Science Foundation of China (Nos. 30771120 and 81072103).

\section{REFERENCES}

[1] Y. Takahashi, M. Suzuki and T. Fukusato, "Plexiform Angiomyxoid Myofibroblastic Tumor of the Stomach," World Journal of Gastroenterology, Vol. 16, No. 23, 2010, pp. 2835-2840. doi:10.3748/wjg.v16.i23.2835

[2] Y. Takahashi, S. Shimizu, T. Ishida, K. Aita, S. Toida, T. Fukusato and S. Mori, "Plexiform Angiomyxoid Myofibroblastic Tumor of the Stomach," American Journal of Surgical Pathology, Vol. 31, No. 5, 2007, pp. 724-728. doi:10.1097/01.pas.0000213448.54643.2f

[3] A. Kim, Y. K. Bae, H. C. Shin and J. H. Choi, "Plexiform Angiomyxoid Myofibroblastic Tumor of the Stomach: A Case Report,” Journal of Korean Medical Science, Vol. 26, No. 11, 2011, pp. 1508-1511. doi:10.3346/jkms.2011.26.11.1508

[4] L. M. Wang and R. Chetty, "Selected Unusual Tumors of the Stomach: A Review," International Journal of Surgical Pathology, Vol. 20, No. 1, 2012, pp. 5-14. doi:10.1177/1066896911429300

[5] M. Miettinen, H. R. Makhlouf, L. H. Sobin and J. Lasota, "Plexiform Fibromyxoma: A Distinctive Benign Gastric Antral Neoplasm Not to Be Confused with a Myxoid GIST,” American Journal of Surgical Pathology, Vol. 33, No. 11, 2009, pp. 1624-1632. doi:10.1097/PAS.0b013e3181ae666a

[6] M. Miettinen, C. D. Fletcher, L. G. Kindblom and T. WM, "Mesenchymal Tumours of the Stomach," In: F. T. Bosman, F. Carneiro, R. Hruban and N. D. Teise, Eds., WHO Classification of Tumours of the Digestive System, IARC, Lyon, 2010, pp. 74-79. 